\title{
A simple scoring system predicted clinical progression in HIV patients receiving highly active antiretroviral therapy
}

Lundgren JD, Mocroft A, Gatell JM, et al, for the EuroSIDA Study Group. A clinically prognostic scoring system for patients receiving highly active antiretroviral therapy: results from the EuroSIDA study. J Infect Dis 2002 Jan 15;185:178-87.

\section{QUESTION: In patients with HIV who are receiving highly active antiretroviral therapy (HAART), does a scoring system predict clinical progression?}

\section{Design}

Prognostic scoring system developed in 1 derivation cohort and validated in 2 cohorts of patients.

Sources of funding: European Commission BIOMED 1; Fifth

Framework Program, GlaxoSmithKline,

Roche, and Boehringer

Ingelheim; Swiss

Federal Office for

Education and Science.

For correspondence:

Dr J D Lundgren,

Hoidoure University

Hospital, Hoidoore

Denmark.jdl@cphiv.dk.

\section{Setting}

60 centres in Europe and Israel.

\section{Patients}

2027 patients (median age $37 \mathrm{y}$ ) formed the derivation set: inception cohort of participants in the EuroSIDA study who were $\geqslant 16$ years of age and had started a protease inhibitor or non-nucleoside reverse-transcriptase inhibitor as part of a HAART antiretroviral regimen and had CD4 cell counts and viral loads measured within 6

\section{COMMENTARY}

Despite the success of HAART in reversing the immunosuppression seen in patients infected with HIV, some patients receiving HAART still have poor outcomes. To quantify the risk for a poor outcome, Lundgren et al developed a point system based on CD4 $\mathrm{T}$ cell numbers, viral load, haemoglobin concentration, and previous diagnosis of "severe" AIDS. (Readers should note that there is a typographic error in the description of the scoring system at the bottom of page 181; the cutoffs for the scoring of viral load are $<500 \mathrm{copies} / \mathrm{ml}, 500$ to $9999 \mathrm{copies} / \mathrm{ml}$, and $\geqslant 10000 \mathrm{copies} / \mathrm{ml}$.) The risk for progression varied from $1 / 100$ person years with a score of 0 , to about $11 / 100$ person years with a score of 6 , to $>100 / 100$ person years with a score of $\geqslant 12$. The strengths of this system are its simplicity, the fact that similar markers were used to predict progression in patients before use of HAART, the large numbers of patients used for both the derivation and validation sets, and the fact that intuitively the components of the prediction guide seem to be relatively important measures.

Despite these advantages, I suspect that most clinicians will not calculate a score but rather will use the individual variables to guide decision making because the study offers no insight into how a calculated score would change clinical practice. Furthermore, several recently recognised factors were not included in the derivation of the guide: co-infection with hepatitis viruses (especially hepatitis $\mathrm{C}$ and hepatitis G), ${ }^{1}$ viral resistance, achieving a viral load $<50 \mathrm{copies} / \mathrm{ml}{ }^{2}$ and transient increases in viral load (referred to as "blips")."

This study will perhaps best be used as a starting point for a clinical trial in which patients with a high score are randomly allocated to 1 of several groups: continuing current treatment, switching to an alternative HAART regimen, using recombinant erythropoietin, or utilising a "drug holiday", usually referred to as a structured treatment interruption.

Bradley Bender, MD Veterans Affairs Medical Center Gainesville, Florida, USA

1 Tillmann HL, Heiken H, Knapik-Botor A, et al. Infection with GB virus C and reduced mortality among HIV-infected patients. N Engl J Med 2001;345:715-24.

2 Durant J, Clevenbergh P, Halfon P, et al. Drug-resistance genotyping in HIV-1 therapy: the VIRADAPT randomised controlled trial. Lancet 1999;353:2195-9.

3 Havlir D, Bassett R, DeGruttola V, et al. Are episodes of transient viremia ("blips" in HIV RNA) predictive of virologic failure in heavily treatment-experienced patients? [Abstract]. 9th Conference on Retroviruses and Opportunistic Infections, Seattle, WA, 24-28 Feb 2002. months before starting HAART and $\geqslant 1$ measurement after starting HAART. 2 cohorts of patients formed the validation set: 1946 patients in the EuroSIDA study who started HAART before recruitment to the study and 1442 patients from a clinic in Barcelona who had previously started HAART.

\section{Description of prediction guide}

Cox proportional hazards models were used to determine factors associated with clinical progression. The final prediction model comprised the latest CD4 cell count, viral load, haemoglobin concentration, and clinical status at the start of HAART (no AIDS, severe AIDS [progressive multifocal leucoencephalopathy and nonHodgkin's lymphoma], or other AIDS diagnosis). For CD4 cell counts, the cut points were 50 and 200 cells $/ \mathrm{mm}^{3}$; for viral loads, the cut points were 500 and $10000 \mathrm{HIV}$-RNA copies/ml. The normal haemoglobin concentration was defined as $>14 \mathrm{~g} / \mathrm{dl}$ for men and $12 \mathrm{~g} / \mathrm{dl}$ for women; severe anaemia was defined as $<8 \mathrm{~g}$ /dl for men and women. Logarithms of the relative hazards were used to derive a score for the variables with independent association with the risk for clinical progression. The score ranged from 0 to 17 points.

\section{Main outcome measure}

Clinical progression (new diagnosis of an AIDS defining event or death).

\section{Main results}

Among patients in the derivation cohort, 200 patients $(9.9 \%$ ) clinically progressed (death $30 \%$, first AIDS defining illness $47 \%$, and new AIDS defining illness $24 \%)$. For a 1 unit increase in score, a mean increase of $38 \%$ (95\% CI 33\% to 43\%) occurred in risk for clinical progression. The incidence of clinical progression was 3.9/100 person years. In the EuroSIDA and Barcelona validation cohorts, the incidence of clinical progression was 4.2 and 4.6 per 100 person years, respectively. A single point increase in score was associated with an increased risk for clinical progression of about $40 \%$ among the 3 cohorts.

\section{Conclusions}

In patients with HIV who are receiving highly active antiretroviral therapy, a scoring system based on the latest CD4 cell count, viral load, and haemoglobin concentration and a previous severe AIDS diagnosis was predictive of clinical progression. The risk for disease progression of a 1 unit increase in score was about $40 \%$. 